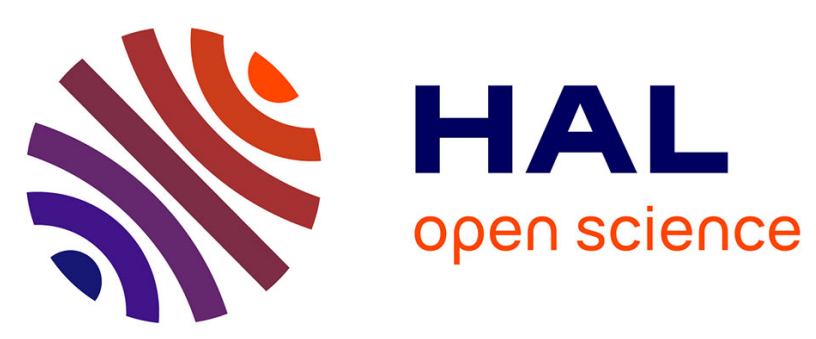

\title{
Line shape in a free-jet hypersonic expansion investigated by cavity ring-down spectroscopy and computational fluid dynamics
}

Nicolas Suas-David, Vinayak Kulkarni, Abdessamad Benidar, Samir Kassi, Robert Georges

\section{To cite this version:}

Nicolas Suas-David, Vinayak Kulkarni, Abdessamad Benidar, Samir Kassi, Robert Georges. Line shape in a free-jet hypersonic expansion investigated by cavity ring-down spectroscopy and computational fluid dynamics. Chemical Physics Letters, 2016, 659, pp.209-215. 10.1016/j.cplett.2016.06.082 . hal-01358139

\section{HAL Id: hal-01358139 \\ https://hal.science/hal-01358139}

Submitted on 17 Nov 2016

HAL is a multi-disciplinary open access archive for the deposit and dissemination of scientific research documents, whether they are published or not. The documents may come from teaching and research institutions in France or abroad, or from public or private research centers.
L'archive ouverte pluridisciplinaire $\mathbf{H A L}$, est destinée au dépôt et à la diffusion de documents scientifiques de niveau recherche, publiés ou non, émanant des établissements d'enseignement et de recherche français ou étrangers, des laboratoires publics ou privés. 


\section{Line Shape in a Free-jet Hypersonic Expansion Investigated by Cavity Ring-down Spectroscopy and Computational Fluid Dynamics}

Nicolas Suas-David ${ }^{\mathrm{a}}$, Vinayak Kulkarni ${ }^{\mathrm{a}, \mathrm{b}, *}$, Abdessamad Benidar ${ }^{\mathrm{a}}$, Samir Kassic and Robert Georges $^{\text {a }}$

anstitut de Physique de Rennes, UMR 6251, Campus de Beaulieu, Bât 11C, Université de Rennes 1/CNRS, F-35042 Rennes Cedex, France

${ }^{\mathrm{b}}$ Department of Mechanical Engineering, Indian Institute of Technology Guwahati, Guwahati, India Pin :

781039.

'Université Grenoble Alpes, LIPhy, F-38000 Grenoble, France and CNRS, LIPhy, F-38000 Grenoble,

France

Manuscript Submitted to the Journal

Chemical Physics Letters

* Correspondence:

Vinayak N Kulkarni

Email Address: vinayak@iitg.ernet.in

November 2015 


\begin{abstract}
Experiments are carried out for spectroscopic studies using hypersonic jet of carbon monoxide seeded in argon as a carrier gas. Probing of this jet using cavity ring-down spectroscopy revealed a double peak structure for various absorption lines. Flow field simulation using computational fluid dynamics is used to understand the shape of such lines integrated over line of sight. Absorption contribution from warmer non-isentropic part of the jet, owing to its transverse velocity variation, is found responsible for those line shapes.
\end{abstract}

\title{
1. Introduction
}

Experimental facilities with supersonic or hypersonic free expansion of gases are widely used as tools for molecular spectroscopic studies. Such jets are characterized by supersaturated gaseous conditions with low rotational temperatures which simplify the structure of molecular spectra. Therefore, steady expanded jets or pulsed jets are conventionally used to attain desired levels of cooling. Understanding the line shape for a particular transition has been one of the major objectives of free-jet based spectroscopic studies. Among such fundamental studies, Veeken and Reuss considered free expansion of ammonia initially to determine the rotational temperature and onwards to estimate the number density [1]. There, anomalous line shape with double peak structure was attributed to condensation occurring preferentially in the allegedly denser axial region. Similar line shape with central shallow dip was also noticed by Mizugai et al. [2]. Snels and Baldacchini [3] conducted experiments with ammonia using diode laser and found non-Gaussian line shape with central dip for rotation-vibration lines of $v_{2}$ band. Clustering at the axis was invoked for those line shapes showing dependence with probing distance from the nozzle exit and the stagnation pressure of the jet. In a similar study, apparent double peak line structure was attributed to clustering by Bajaj et al. [4]. Possibility of clustering was also reported in the supersonic jet of $\mathrm{N}_{2} \mathrm{O}$ [5] and acetylene [6].

While one of the explanations for anomalous line shapes is the formation of clusters in the neighborhood of jet axis, other explanations are also available in the literature. Gaveau et al. numerically predicted shape and width of absorption lines in case of CO-He free-jet for $\mathrm{P}(1)$ and $\mathrm{P}(2)$ lines [7]. In their study, shape of the absorption lines was found to be dependent on the variation of density and temperature in the jet. However the effect of jet boundary was not considered in that study. Campbell et al. found that 'Doppler dephasing' of rotational lines is responsible for the peculiar line shape observed in their microwave study of OCS and rare gas/OCS complexes [8]. This explanation was found non-unique by Lovas and Suenram for the supersonic jet of OCS seeded in various rare gases [9]. Besides, 'doubling' of line shape was accounted for loss of density due to clustering of OCS with carrier gas. Nevertheless, in 
their collaborative efforts, Campbell and Lovas finally concluded that density and temperature gradients along the laser beam are responsible for double peaked lines [10]. Ramos et al. disregarded condensation of nitrogen and attributed the observed double peak structure of lines to inhomogeneous line broadening due to strong temperature and density gradients within the silent zone, along the line of sight [11].

Thus diversity can be seen in the literature for explaining line shape with two peaks. In some of the reported findings, it has been noticed that, the observations used for justification of clustering do not exclusively conclude the clustering. Besides, flow field gradients can also be led responsible for such observations. However, it has also been felt that the studies, which account for flow field along the laser beam for the anomalous line shape, need detailed investigations to mark specific region of the flow and the flow property responsible for such line shape. In view of this, spectroscopic measurements are carried out for hypersonic jet of carbon monoxide and argon using cavity ring-down spectroscopy (CRDS). These studies are continuation of the earlier findings of Louviot et al. [12] where as well double peak structure of absorption lines had been observed for carbon monoxide in CRDS based measurements. Therefore present investigations specifically deal with analysis of absorption line shapes for line of sight spectroscopic measurements. Numerical simulations are also carried out to get the simulated lines. Efforts are then extended to correlate the experimental and simulated lines with structure of the free-jet. Details of the experimental setup, numerical simulations and reasoning for the anomalous line shape are given in the following sections.

\section{Experimental and numerical methodology}

Experiments are carried out in an experimental facility having a High Enthalpy Source (HES) connected to a vacuum chamber [12, 13]. In the present studies, carbon monoxide (Air Liquide, 99.998\% purity) is used as the seeding gas (5\% by mass) and argon (Air Liquide, $99.998 \%$ purity) as the carrier gas (95\% by mass). Mass flow rate of this mixture is regulated while flowing through HES where it is heated by passing 80 A current through graphite. A circular nozzle of $2 \mathrm{~mm}$ diameter is used as a throat for expansion of the gas in the vacuum chamber. This chamber is evacuated to the pressure of $11 \pm 1 \mathrm{~Pa}$ so as to establish flow field known as a Campargue-type expansion [14]. Expansion of jet is probed using high sensitive cw-CRD spectrometer. Telecom grade fibered Distributed Feedback (DFB) diode lasers of power 10-20 mW are used to act as light source. Each of these lasers covers about $7 \mathrm{~nm}$ of wavelength $\left(\sim 30 \mathrm{~cm}^{-1}\right)$. For this, their temperatures are adjusted between -10 to $60^{\circ} \mathrm{C}$ with the help of in-house developed proportional integral differential (PID) stabilizer. From the total light emitted by the laser, $10 \%$ is transferred to wavemeter (Burleigh WA-1600) and 90\% is introduced into the optical cavity. This high finesse cavity ( $\mathrm{F}>200000)$ consists of two high reflectivity (>99.9987\%), plano-concave (1000 mm 
radius) mirrors which are separated by about $800 \mathrm{~mm}$. Mode matching between laser beam and TEM $\mathrm{M0}_{00}$ mode of cavity is achieved using single lens and two steering mirrors. Cavity length is modulated by mounting the output mirror on a piezoelectrical transducer in order to set resonance with laser frequency. At the resonance, acousto-optical modulator (AOM) gets switched off, owing to which photons evolve freely inside the cavity. Ring-down time is then obtained using an exponential fit of the intensity measured by a photodiode. Spectroscopic measurements are carried out at locations which are $5 \mathrm{~mm}, 20 \mathrm{~mm}$ and 40 $\mathrm{mm}$ downstream of the nozzle exit. These locations would be referred here onwards as $\mathrm{X} 1, \mathrm{X} 2$ and $\mathrm{X} 3$ respectively. It should be noted here that, the location of Mach disk for present experimental conditions is about $16 \mathrm{~cm}$ from nozzle exit. Therefore these axial probing stations are about $1 / 32^{\text {th }}, 1 / 8^{\text {th }}$ and $1 / 4^{\text {th }}$ of the Mach disk location. These line of sight measurements are always ensured in the direction perpendicular to the jet axis. Experimental data is recorded using a LabVIEW based data acquisition system for the spectral range of carbon monoxide as [6220-6320] $\mathrm{cm}^{-1}$ corresponding to the $3 \leftarrow 0$ vibrational band.

Two numerical techniques, computational fluid dynamics (CFD) and method of characteristics (MOC), are employed to predict flow properties in the jet. These predictions form inputs for obtaining two synthetic absorption lines. Among these numerical techniques, initially, commercial CFD solver Fluent of ANSYS-15 [15] is used to visualize and understand the structure of hypersonic free-jet. This viscous flow solver considers mass, momentum and energy conservation equations to simulate the flow from reservoir till $50 \mathrm{~mm}$ downstream of the orifice. Beyond this distance, the Knudsen number $\left(\mathrm{K}_{\mathrm{n}}\right)$, which can be defined as the ratio between the mean free path of the expanding molecules and the nozzle diameter, becomes too high. As a result of this, Fluent solver remains invalid due to break down of continuum assumption necessary for using Navier-Stokes equation. It has also been noticed from the experimental conditions that the Knudsen number for present case varies between 0.2 (core) and 0.6 (shear layer / jet boundary) which is still far from the free molecular regime $\left(K_{n}>10\right)$. Thus, using this computational technique, it is possible to analyze the flow in all parts of the jet which would later be helpful to construct the absorption line along entire line of sight. In view of this, two dimensional (2D) axi-symmetric viscous simulations are carried using mixture properties and assumption of perfect gas. Density based solver and Sutherland's law for viscosity are opted for these computations. Reservoir conditions $\left(\mathrm{P}_{0}=133.32 \mathrm{kPa}\right.$ and $\mathrm{T}_{0}=1300 \mathrm{~K}$ ) are provided at the inlet of the fluid domain. Supersonic flow boundary condition with chamber pressure is implemented at the outlet. Converged steady state results are considered for further analysis. After viscous flow simulations using CFD, MOC based fit has been initially used to get the axial variation of Mach number. Axial temperature of the flow is then recovered using law of energy conservation for adiabatic expansion. Other flow variables are also obtained from isentropic relations. Then Eq. 1 is used to estimate off-axial variation of density in the silent zone [16]. 


$$
\rho(z, y)=\rho(z, 0)\left\{\cos ^{2}[\theta(z, y)]\right\}\left\{\cos ^{2}[\pi \theta(z, y) / 2 \phi]\right\}
$$

Here, $\rho(z, y)$ is off axial density evaluated from the axial counterpart $[\rho(z, 0)]$ and angle made by velocity vector with jet axis $(\theta)$. Value of $\phi$, given by Ashkenas and Sherman [16] is used here. This equation is the best fit for MOC based prediction hence it is valid only in the isentropic core of jet [16]. Therefore, boundary of isentropic core is mandatory input for this approach to construct the computational domain. Results obtained from CFD simulations are considered here to provide this valuable input. Thus obtained off-axial density and known reservoir density are used to evaluate local temperature using isentropic relation. Mach number of any location is then obtained from isentropic relation with the help of local and stagnation temperatures. Velocity is computed using Mach number and temperature.

Flow field information received from CFD based 2D axi-symmetric simulations is used to build the entire three dimensional (3D) jet. Besides, only 3D inviscid core is constructed using similar information given by MOC. These transformations help to obtain the absorption line shape for entire line of sight (CFD) and for limited sight (MOC) at any laser probing station. At a given probing station, laser beam of $1 \mathrm{~mm}$ diameter with Gaussian intensity distribution and zero divergence is introduced in such 3D domains. Since the chosen mesh for computations has length scale of the order $0.02 \mathrm{~mm}$, it ensures participation of sufficient number of cells (identified by $x, y$ and $z$ co-ordinates) in the laser beam for predicting the absorption line shape. Then, the flow properties viz. component of velocity in the line of sight, density and temperature are used to get the synthetic line from CFD and MOC. Equation 2 is used for calculation of absorption coefficient integrated along the line of sight.

$\alpha(\widetilde{v})=\sum_{z}\left\{\sum_{x y}\left[n_{x y z} \bar{\sigma}_{i j}\left(T_{x y z}\right) g_{D}\left(\tilde{v}-\widetilde{v}_{i j}^{\prime}\right) \frac{l(z)}{L}\left(\exp \left(-\frac{x^{2}+y^{2}}{w_{0}^{2}}\right)\right)^{2} \frac{1}{\sum_{x y}\left(\exp \left(-\frac{x^{2}+y^{2}}{w_{0}^{2}}\right)\right)^{2}}\right]\right\}$

Here $\mathrm{x}, \mathrm{y}$ and $\mathrm{z}$ are Cartesian coordinates, $\mathrm{n}_{\mathrm{xyz}}$ is number density (molecule $\mathrm{cm}^{-3}$ ) corresponding to the cell or elemental volume at $x, y$ and $z, \mathrm{~T}(\mathrm{x}, \mathrm{y}, \mathrm{z})$ is temperature $(\mathrm{K})$ of the cell, $\bar{\sigma}_{i j}(T)$ is integrated absorption cross section, $g_{D}$ is Gaussian line shape with thermal Doppler effect (temperature based line broadening) and convective Doppler effect (transverse velocity based line broadening), $\widetilde{v}$ is wave-number $\left(\mathrm{cm}^{-1}\right), \mathrm{i}$ and $\mathrm{j}$ are lower and upper states of the spectroscopic transition, $l(z)$ is length scale of a cell along line of sight $(\mathrm{cm})$, $\mathrm{L}$ is length of cavity $(\mathrm{cm})$ and $\mathrm{w}_{0}$ is waist of laser beam $(\mathrm{cm})$. The integrated absorption cross section is estimated here as 


$$
\bar{\sigma}_{i j}(T)=\bar{\sigma}_{i j}\left(T_{r e f}\right) \frac{Q\left(T_{r e f}\right)}{Q(T)} \exp \left(\frac{E_{i}}{k_{B} T_{r e f}}-\frac{E_{i}}{k_{B} T}\right)\left[\frac{1-\exp \left(-\frac{\tilde{v}_{i j}}{k_{B} T}\right)}{1-\exp \left(-\frac{\widetilde{v}_{i j}}{k_{B} T_{r e f}}\right)}\right]
$$

where, $\mathrm{Q}$ is partition function, $\mathrm{E}$ is energy level $\left(\mathrm{cm}^{-1}\right)$ and $\mathrm{k}_{\mathrm{B}}$ is Boltzmann constant $\left(0.69503 \mathrm{~cm}^{-1} \mathrm{~K}^{-1}\right)$. Required integrated absorption cross section at $\mathrm{T}_{\text {ref }}(296 \mathrm{~K})$ is extracted from the 2012 HITRAN database [17]. Equilibrium partition function [18] is considered here since the flow simulation has assumption of equilibrium between translational, rotational and vibrational modes of temperature. Gaussian line profile, from Eq. 2, is calculated as,

$$
g_{D}\left(\tilde{v}-\tilde{v}_{i j}^{\prime}\right)=\sqrt{\frac{\ln 2}{\pi}} \frac{1}{\gamma_{D}} \exp \left(-\ln (2)\left[\frac{\tilde{v}-\tilde{v}_{i j}^{\prime}}{\gamma_{D}}\right]^{2}\right)
$$

Some of the terms of this equation are,

$$
\begin{gathered}
\widetilde{v}_{i j}^{\prime}=\tilde{v}_{i j}\left(1 \pm \frac{v_{\perp}}{c}\right) \\
\gamma_{D}=\sqrt{\ln 2} \frac{v_{m p}}{c} \tilde{v}_{i j} \\
v_{m p}=\sqrt{\frac{2 R T}{M}}
\end{gathered}
$$

Here,

$\gamma_{D}$ is half width at half maximum for thermal broadening $\left(\mathrm{cm}^{-1}\right)$, $v_{\perp}$ is transverse velocity or component of velocity in the line of sight $(\mathrm{m} / \mathrm{s})$, c is Speed of light $(\mathrm{m} / \mathrm{s})$, $v_{m p}$ is most probable speed $(\mathrm{m} / \mathrm{s}), \mathrm{R}$ is Universal gas constant $(\mathrm{J} / \mathrm{mol} \mathrm{K})$ and $\mathrm{M}$ is molecular weight $(\mathrm{kg} / \mathrm{mol})$. Prediction of line shape using this methodology is advantageous since it gives absorption line shape for any laser length at a given axial location. Thus it helps in understanding contribution of each region of jet in building the integrated absorption line.

\section{Results and Discussion}

Mach number, transverse velocity, temperature and density variations in the hypersonic free-jet, obtained from CFD simulations, are shown in Fig. 1. Jet probing stations, considered during experiments, and experimentally recorded $\mathrm{P}(1)$ lines at those stations, are shown in the transverse velocity contours. Figure 1 helps to understand expansion of the gas and thus evolved jet structure encountered by the laser 
during measurement. In the expansion process, gas attains sonic state at the exit of nozzle from reservoir conditions and then expands to very low pressure in the isentropic core. Mach disk limits expansion of gas in the axial direction but present simulation is terminated before the Mach disk so as to analyze the flow field in the neighborhood of nozzle exit. Compression waves limit expansion of the gas in transverse direction. Subsequently, there is jet boundary or shear layer in the transverse direction which is portion of the jet decelerated by static residual gas in the chamber. However chamber fluid also gets entrained while decelerating the jet. Thus, it is evident here that, laser at any axial location interacts with four regions of the jet viz. near axis flow region with negligible transverse velocity (A), off axial flow with finite transverse velocity (B), region of compression waves (C) and the jet boundary (D). Colder regions A and $\mathrm{B}$ are necessarily isentropic while warmer regions $\mathrm{C}$ and $\mathrm{D}$ are non-isentropic in nature. Only transverse velocity variation is prominent in the transition from regions $A$ to $B$ while there is marginal change in any other flow property. Boundary between regions B and C is characterized by decrement of Mach number. Sufficient increment in translational temperature would be evidenced while transiting from region C to D. Representative small volume from regions A to D, for probing station X3, are highlighted here using numbers 1 to 4 respectively. This representation is thought to be useful to get the CFD based line shape at these individual stations and onwards to visualize the process of integration of all such local lines in getting the complete integrated line shape. Here, region 1 is a cylinder of diameter $1 \mathrm{~mm}$ (same as laser beam) and length $10 \mathrm{~mm}$ along the laser beam with its geometric center on the jet axis. Regions 2-4 are also circular cylinders of same diameter but of length $5 \mathrm{~mm}$ on either side of jet axis. They have their geometric centers at a distance of 10,20 and $30 \mathrm{~mm}$ respectively from jet axis. Probing station X3 is chosen for demonstration since it is comprised of sufficiently thick regions (A to D) due to which the representative volumes (1 to 4 ) are of equal and adequate sizes and are well separated. But these specifications of representative small volumes ( 1 to 4 ) should be dependent on axial probing station since thickness of regions A to D vary in the flow direction. For example, dimension of jet, along the laser, is very small at $5 \mathrm{~mm}$ from nozzle exit (X1). Here jet would be comprised of comparatively larger sized regions A and B with negligible thicknesses of regions $\mathrm{C}$ and $\mathrm{D}$. Due to change in sizes of regions $\mathrm{A}$ to $\mathrm{D}$ in axial direction, experimentally obtained $\mathrm{P}(1)$ lines at different probing stations (Fig. 1) are expected to have differential contributions from these regions. Apart from this, since present experiments are conducted for high stagnation temperature conditions, it has led to strong thermal non-equilibrium [12] in the jet. Therefore, clustering of carbon monoxide is disregarded due to freezing of vibrational temperature in the expansion process. These facts strengthen the possibility of correlation between observed double peaked absorption lines with the jet structure.

Figure 2 shows the contribution of each region in building $\mathrm{P}(1)$ line for location X3. Process of integration is illustrated in Fig. 2(a) while Fig. 2(b) gives the outcome of this process of integration 
associated with construction of experimentally obtained $\mathrm{P}(1)$ line. Vibration-rotation band for representative small volumes (1 to 4 ) is on the left hand side of Fig. 2(b) along with the highlighted P(1) line of that region. In those highlights, only region 1 has $\mathrm{P}(1)$ line with single Gaussian shape while other regions (2-4) show two discretely spaced Gaussians. It is mainly due to discrete appearance of these regions in the line of sight. Right hand side of Fig. 2 (b) (I-IV) shows integrated P(1) line from CFD and MOC based computations where this integration starts from axis of the jet. This integration process accounts for four regions (A to D) for CFD based P(1) line but it accounts for only two regions (A and B) in case of MOC based $\mathrm{P}(1)$ line. While plotting these $\mathrm{P}(1)$ lines, intensities are normalized with respective peak values. It is clear from first part of Fig. 2 (b) (I) that there is no central dip in P(1) line for region 1 in both the simulation techniques. This Gaussian shape can be expected in the axial region of the jet since these numerical techniques do not account for clustering as per their formulations. Further, MOC based simulation does not predict double peak structure of $\mathrm{P}(1)$ line in the second phase of integration for complete isentropic core or silent zone of the flow (II). Unlike MOC, shallow dip can be noticed in the CFD based synthetic P(1) line for this axial and off-axial parts of the flow (II) which are predominantly inviscid. Major reasons for this computational artifact are the governing equations for 2D axi-symmetric fluid flows which hold singularity at the axis and the associated computational cost required to overcome the same. Such artifact can also be noticed in the findings of Mate et al. [19] where density variation close to the axis is not in agreement with the experiments and expected MOC based variation. In case of CFD simulations, double peak structure of $\mathrm{P}(1)$ line becomes prominent in the next integration phase where non-isentropic region 3 is also included (III). Since computations with MOC are not valid in this region, here (III) corresponding MOC based $\mathrm{P}(1)$ line is same as of II and represents contribution of entire isentropic core only. Moreover, $\mathrm{P}(1)$ line is seen to be deeper and wider after accounting for regions like 4 in the process of complete integration along the line of sight (IV). Therefore, at the end of integration in the line of sight (IV), CFD based $\mathrm{P}(1)$ line is compared with the experimentally obtained $\mathrm{P}(1)$ line which as well exhibits same double peak structure. Here also (IV), MOC based P(1) line, which has a plateau at the peak, is same as II and refers to isentropic core. Thus it is evident from this process of integration (IIV) that the isentropic core is not responsible for the double peaked structure in $\mathrm{P}(1)$ line corresponding location X3. Besides this, it is the non-isentropic region of the jet which is responsible for such shape of $\mathrm{P}(1)$ line. For better understanding, variation of flow properties along the line of sight is plotted in Fig. 3 and $\mathrm{P}(1)$ lines of individual regions (1-4), as per their transverse locations, are plotted in Fig. 4. These two figures help in establishing correlation between flow properties in the probing direction with the integrated line shape. Here, intensity of each Gaussian in Fig. 4 is by the virtue of local temperature and density given in Fig. 3. However, displacement of each Gaussian on the frequency axis is attributed to the component of velocity along the line of sight. There is reduction in peak intensity of the local Gaussian of 
isentropic core with increase in distance from the jet axis. Along with this, these Gaussians can be seen to be linearly displaced on the frequency axis due to linear variation of transverse velocity with increase in distance from the axis. This fact has its basis on conical expansion of the jet in the silent zone or diverging streamline pattern in the isentropic core (Fig. 1). Due to such a flow structure, no central dip in the P(1) line can be expected from MOC based computations (Fig. 2 (b), II). However flattening of the peak of such MOC based $\mathrm{P}(1)$ line is dependent on the local diameter of isentropic core (divergence of the flow or convective Doppler effect) and thermal line broadening (Thermal Doppler effect). Further, non-isentropic region of the jet needs to be considered to explain such a line shape. As per Fig. 4, for non-isentropic regions, peak intensity of the local $\mathrm{P}(1)$ line continues to reduce with increase in transverse coordinate, which can be easily explained by warming of the jet in these regions (Fig.3). It also leads to enhanced thermal broadening of local Gaussians. Nevertheless, it is apparent in Fig. 4 that there is loss of linearity in displacement of these Gaussians on frequency axis with increase in distance from the axis. It is due to reduction in the rate of increase of transverse velocity in the presence of compression waves or due to decrement in transverse velocity in the jet boundary. Such transverse velocity variation is equivalent to deflection of streamline towards the jet axis (Fig. 1). Thus, local Gaussians from non-isentropic region exhibit resistance in their outward shift on frequency axis. Therefore, when this region gets accounted for obtaining integrated $\mathrm{P}(1)$ line, it necessarily adds the thermally broadened Gaussians to the $\mathrm{P}(1)$ line obtained from lone isentropic core of the jet. This mechanism justifies the double peak structure noticed in experimental and synthetic lines in the absence of condensation. Synthetic P(1) line of CFD shows shift in peak at wavenumber of $0.0106 \mathrm{~cm}^{-1}$ for location X3 (Fig. 2 (b), IV). Experimental P(1) line of the same location also shows shift of peak at $0.0080 \mathrm{~cm}^{-1}$ and thus supports the demonstration in getting double peaked $\mathrm{P}(1)$ line. Thus Figs. 2 to 4 clearly illustrate the reasoning behind double peaked $\mathrm{P}(1)$ line for station X3. Similar process can be executed for any other axial station. However, it has been seen that the isentropic core (A and B) is not responsible for double peaked line structure and it is evident from the Fig. $\mathbf{1}$ that the size of the non-isentropic regions (C and D) increase with increase in streamwise distance. Therefore it is expected to have shallow peak near the nozzle exit owing to thin jet boundary. This observation justifies the small dip detected in experimental and synthetic $\mathrm{P}(1)$ lines at X1 location (Fig. 1). It also supports the deeper $\mathrm{P}(1)$ line noticed at X2 and X3 probing stations (Fig. 1). Same jet structure based fluid dynamic reasoning is valid for other absorption lines as shown in Fig. 5. Here the central dip in $\mathrm{P}(2), \mathrm{P}(4)$ and $\mathrm{P}(6)$ lines can be justified in the similar manner. However, $\mathrm{P}(9)$ line shows single peak structure due to the fact that its major contribution is from high temperature fluid from jet boundary which has negligible transverse velocity. 


\section{Conclusion}

Experiments are successfully carried out for probing hypersonic jet of carbon monoxide seeded in argon using cw-CRDS. Viscous simulations are then performed using ANSYS-15 to understand the structure of the jet. Method of characteristics based fits are also employed to simulate the silent zone of the jet. Results from these simulations are then used to demonstrate the construction of absorption lines obtained in the line of sight measurements. In this process, it has been noticed that the isentropic core or silent zone is not responsible for double peaked absorption lines. However, transverse velocity variation in the non-isentropic region is found to add thermally broadened Gaussians for building the double peaked absorption lines. Moreover, height of peak or size of the dip is found dependent on size of this region. Thus present studies are found useful in understanding the absorption line shape and correlating it with structure of the jet. In light of this conclusion, double peaked or anomalous line shapes [20] of monomers reported in the literature are expected to have relevance with the jet profile where explicit evidence of condensation is absent. However, in case of clustering at axis as well, loss of monomers and warmer nonisentropic part of the jet should be accounted for double peaked absorption lines.

\section{Acknowledgements}

Authors would like to acknowledge the financial support from CEFIPRA (Project No. 4905-1 "Kinetics and spectroscopy in extreme environments: Applications to astrophysics and astrochemistry"). RG acknowledges the "Programme National de la Planétologie" (INSU CNRS) for its financial support. RG and SK would like to thank GDR Spec Mo 3152 for the financial support.

\section{References:}

1. K. Veeken and J. Reuss, Appl. Phys. B 34 (1984) 149.

2. Y. Mizugai H. Kuze, H. Jones, and M. Takami, Appl. Phys. B 32, (1983) 43.

3. M. Snels and G. Baldacchini, App. Phys. B 47 (1988) 277.

4. P.N Bajaj, A.K. Talukdar, P. K. Chakraborti and V.B. Kartha, Journal of Molecular Structure, 194 (1989) 117.

5. G. Gimmler and M. Havenith, Journal of Molecular Structure, 599 (2001) 117.

6. K. Didriche, C. Lauzin, P. Macko,W. Lafferty, R. Saykally, and M. Herman, Chem. Phys. Lett. 463 (2008) 345.

7. M.A. Gaveau, D. Boscher and J.P.Martin, Chem. Phys. Lett. 107 (1984) 31. 
8. E. J. Campbell, L. W. Buxton, T. J. Balle, M. R. Keenan and W. H. Flygare, J. Chem. Phys. 74 (1981) 829.

9. F. J. Lovas and R. D. Suenram, J. Chem. Phys. 87 (1987) 2010.

10. E. J. Campbell and F. J. Lovas, Rev. Sci. Instrum., 64 (1993) 2173.

11. A. Ramos, J. Santos, L. Abad, D. Bermejo, V. J. Herrero, and I. Tanarro, J. Raman Spectrosc., 40 (2009) 1249.

12. M. Louviot, N. Suas-David, V. Boudon, R. Georges, M. Rey and S. Kassi, J. Chem. Phys. 142 (2015) 214305.

13. J. Thiévin, R. Georges, S. Carles, A. Benidar, B. Rowe, and J.-P. Champion, J. Quant. Spectrosc. Radiat. Transfer 109 (2008) 2027.

14. H.C.W. Beijerinck, R.J.F. Van Gerwen, E.R.T. Kerstel, J.F.M. Martens, E.J.W. Van Vliembergen, M.R.Th. Smits and G.H. Kaashoek, Campargue-type supersonic beam sources: Absolute intensities, skimmer transmission and scaling laws for mono-atomic gases $\mathrm{He}$, $\mathrm{Ne}$ and Ar; Chemical Physics 96 (1985) 153-173.

15. ANSYS Inc., 2015. ANSYS FLUENT Release 15.0 User Manual, ANSYS Inc.

16. H. Ashkenas, F.S. Sherman, The Structure and Utilization of Supersonic Free Jets in Low Density Wind Tunnels, in: J.H. de Leeuw (Ed.), Rarefied Gas Dynamics, 4th Symp., Academic Press, New York, 1966.

17. L.S. Rothman, I.E.Gordon, Y.Babikov, A.Barbe, D.ChrisBenner, P.F.Bernath, M.Birk, L.Bizzocchi, V.Boudon, L.R.Brown, A.Campargue, K. Chance, E.A.Cohen, L.H.Coudert, V.M.Devi, B.J.Drouin, A.Fayt, J.-M. Flaud, R.R.Gamache, J.J.Harrison, J.-M.Hartmann, C.Hill, J.T.Hodges, D.Jacquemart, A.Jolly, J.Lamouroux, R.J.LeRoy, G.Li, D.A.Long, O.M.Lyulin, C.J.Mackie, S.T.Massie, S.Mikhailenko, H.S.P.Müller, O.V.Naumenko, A.V.Nikitin, J.Orphal, V.Perevalov, A.Perrin, E.R.Polovtseva, C.Richard, M.A.H.Smith, E.Starikova, K.Sung, S. Tashkun, J.Tennyson, G.C.Toon, Vl.G.Tyuterev, G.Wagner, J. Quant. Spectrosc. Radiat. Transfer 130 (2013) 4.

18. R.R. Gamache, S. Kennedy, R. Hawkins, L.S. Rothman, Journal of Molecular Structure 517-518 (2000) 407.

19. B. Mate, I. A. Graur, T. Elizarova, I. Chirokov, G. Tejeda, J.M. Fernandez and S. Montero, J. Fluid Mech. 426 (2001) 77.

20. M. J. Thorpe, F. Adler, K. C. Cossel, M. H.G. de Miranda and J. Ye Chem. Phys. Lett. 468 (2009) 1. 

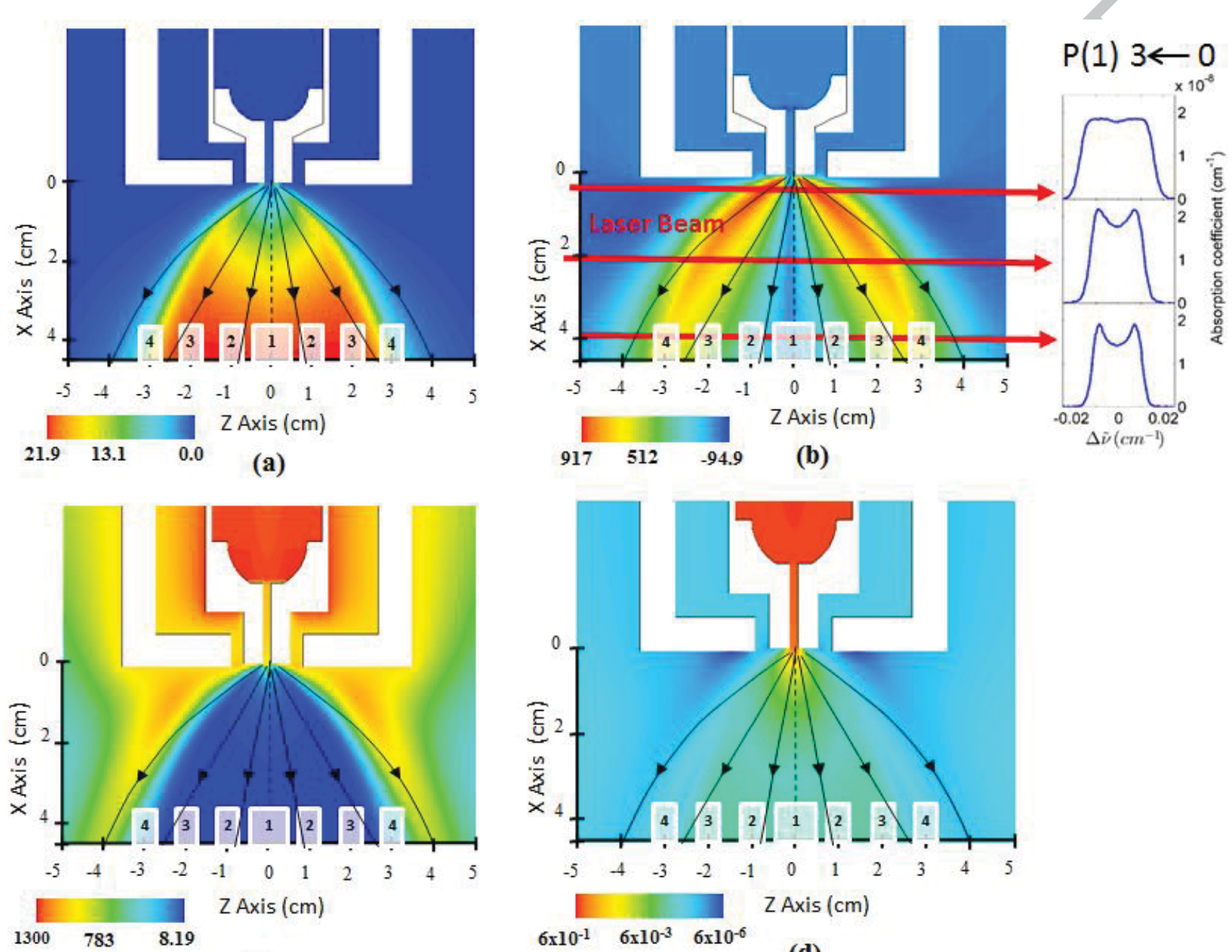

(C)

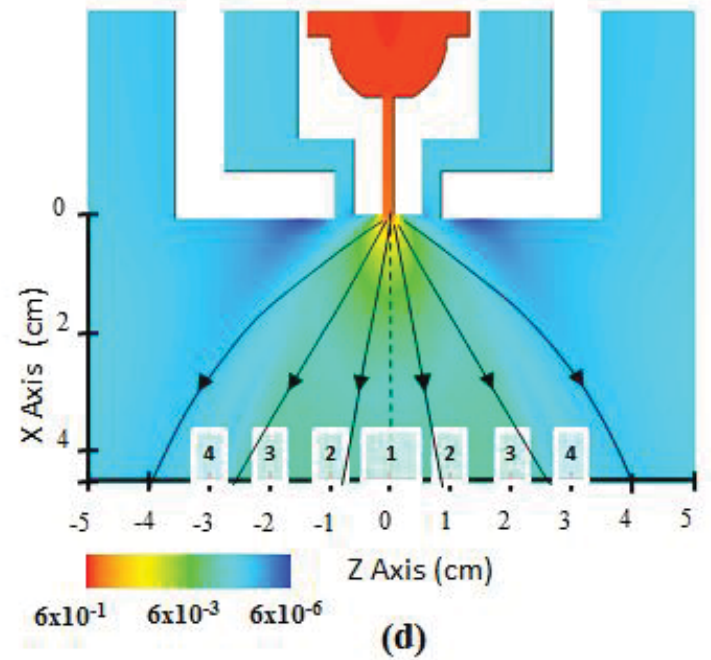

Fig.1 CFD Simulation based variation of flow properties in the jet (a) Mach number, (b) Transverse velocity $(\mathrm{m} / \mathrm{s})(\mathrm{c})$ Temperature $(\mathrm{K})$ and $(\mathrm{d})$ Density $\left(\mathrm{kg} / \mathrm{m}^{3}\right)$. Laser probing stations considered in the experiments and corresponding $\mathrm{P}(1)$ lines are shown in the transverse velocity field (b). 


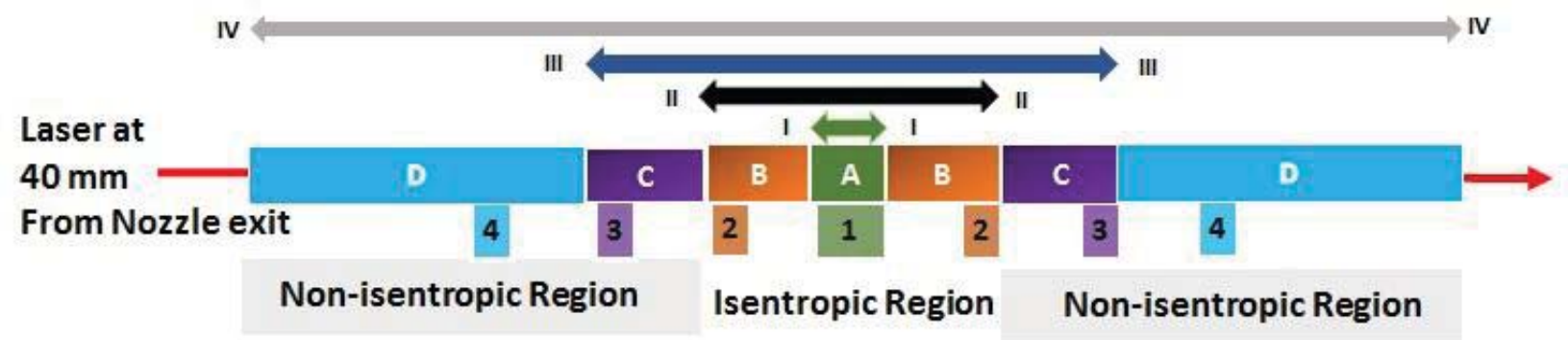

(a)
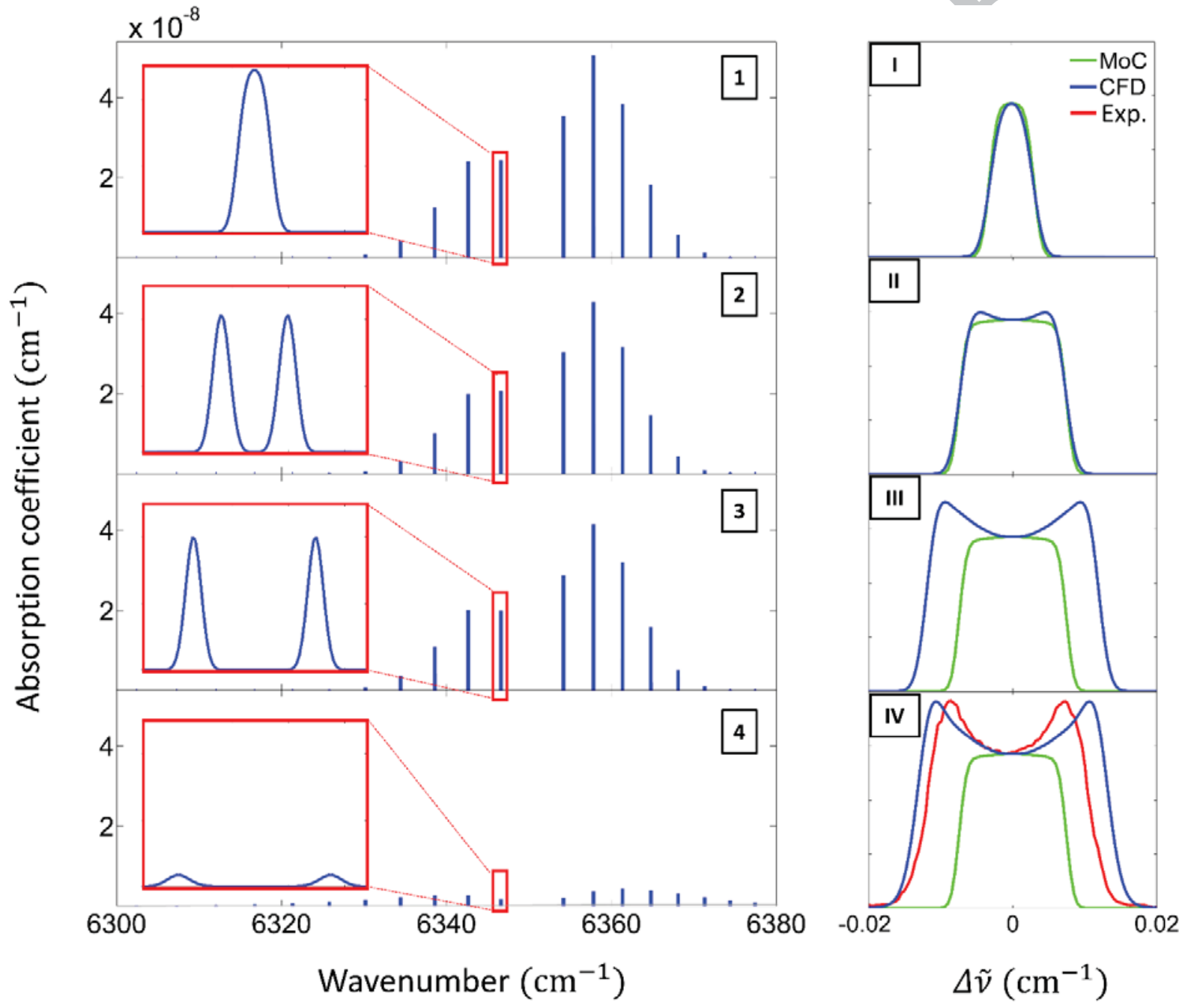

(b)

Fig.2. (a) Process of integration along the line of sight. (b) $3 \leftarrow \mathbf{0}$ vibrational band at X3 (1-4) and corresponding process of obtaining integrated $\mathrm{P}(1)$ line from simulations along with its comparison with experiment (I-IV). 


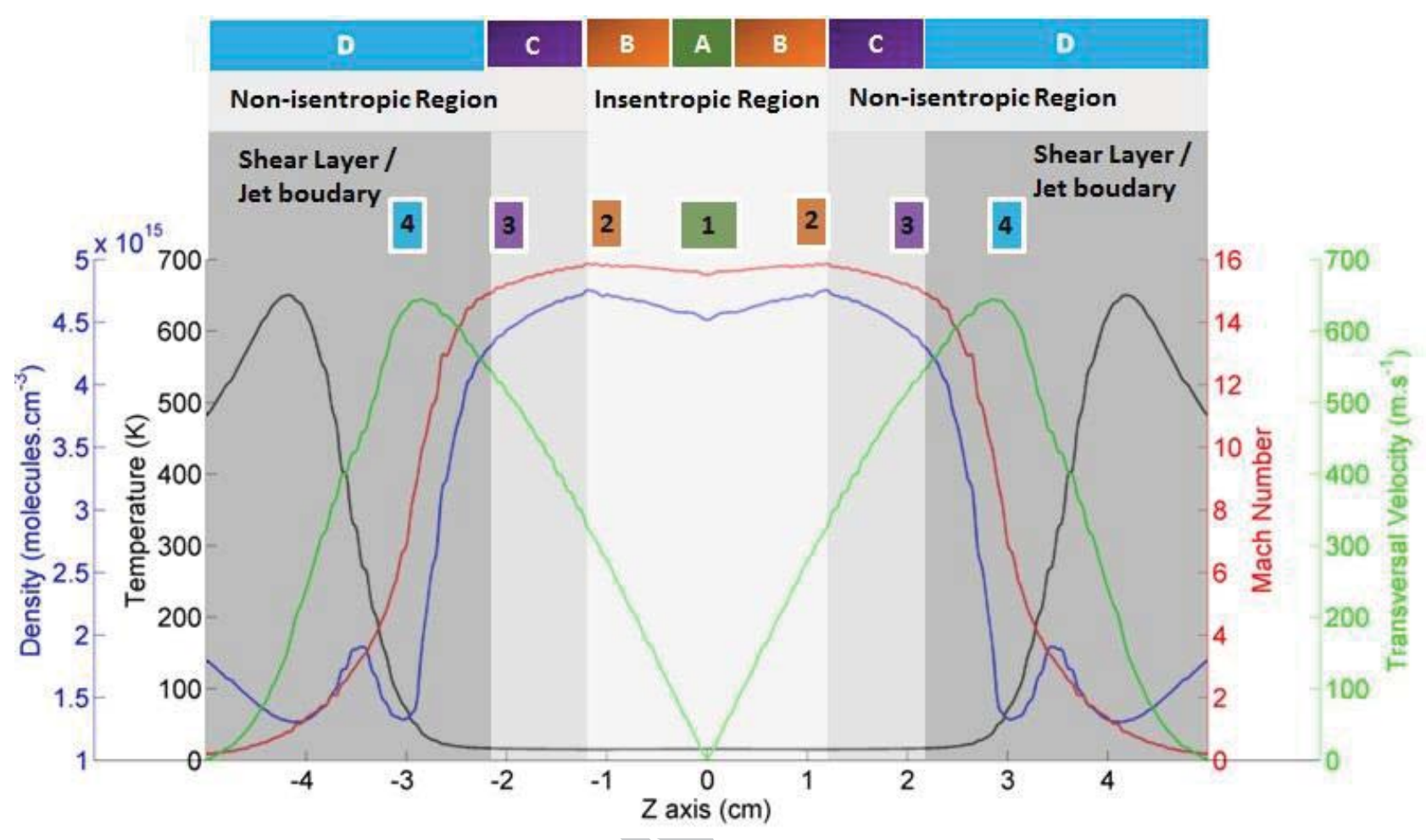

Fig.3 Flow properties obtained from CFD along the line of sight at X3. 


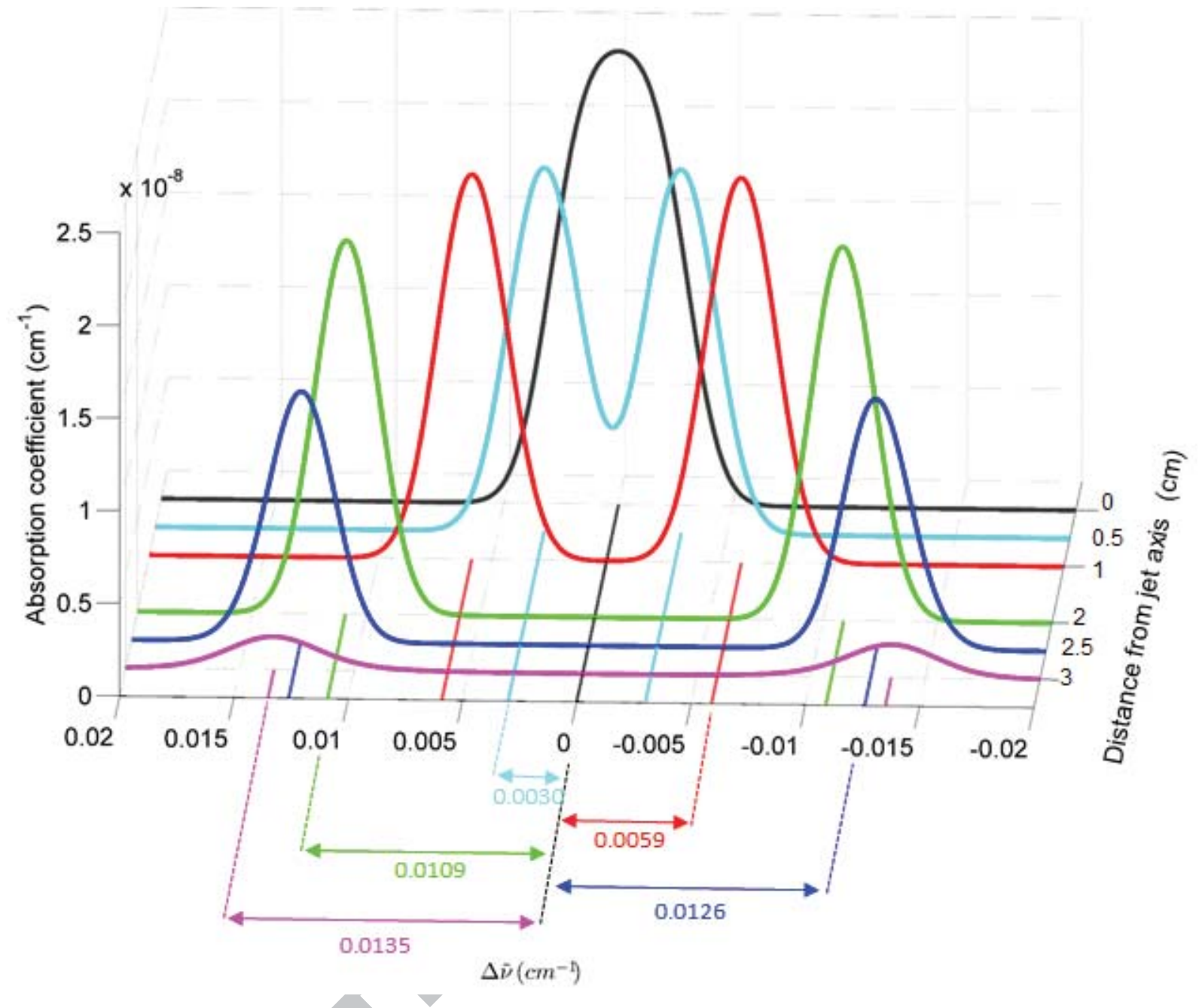

Fig.4 Illustration of building of the integrated P(1) line at X3 using the local Gaussian distributions. 


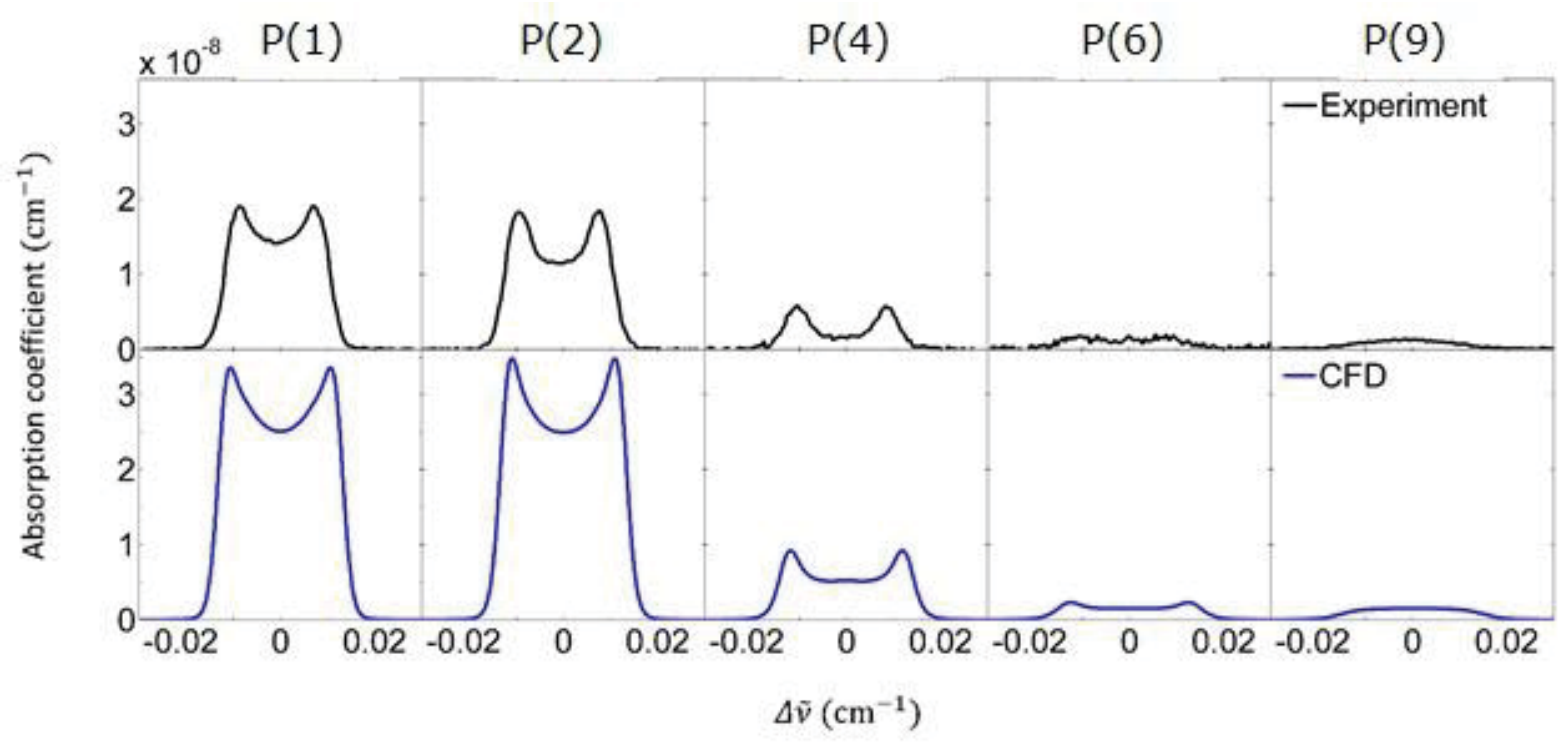

Fig.5 Comparison of simulated and experimental lines at X3. 
Graphical abstract

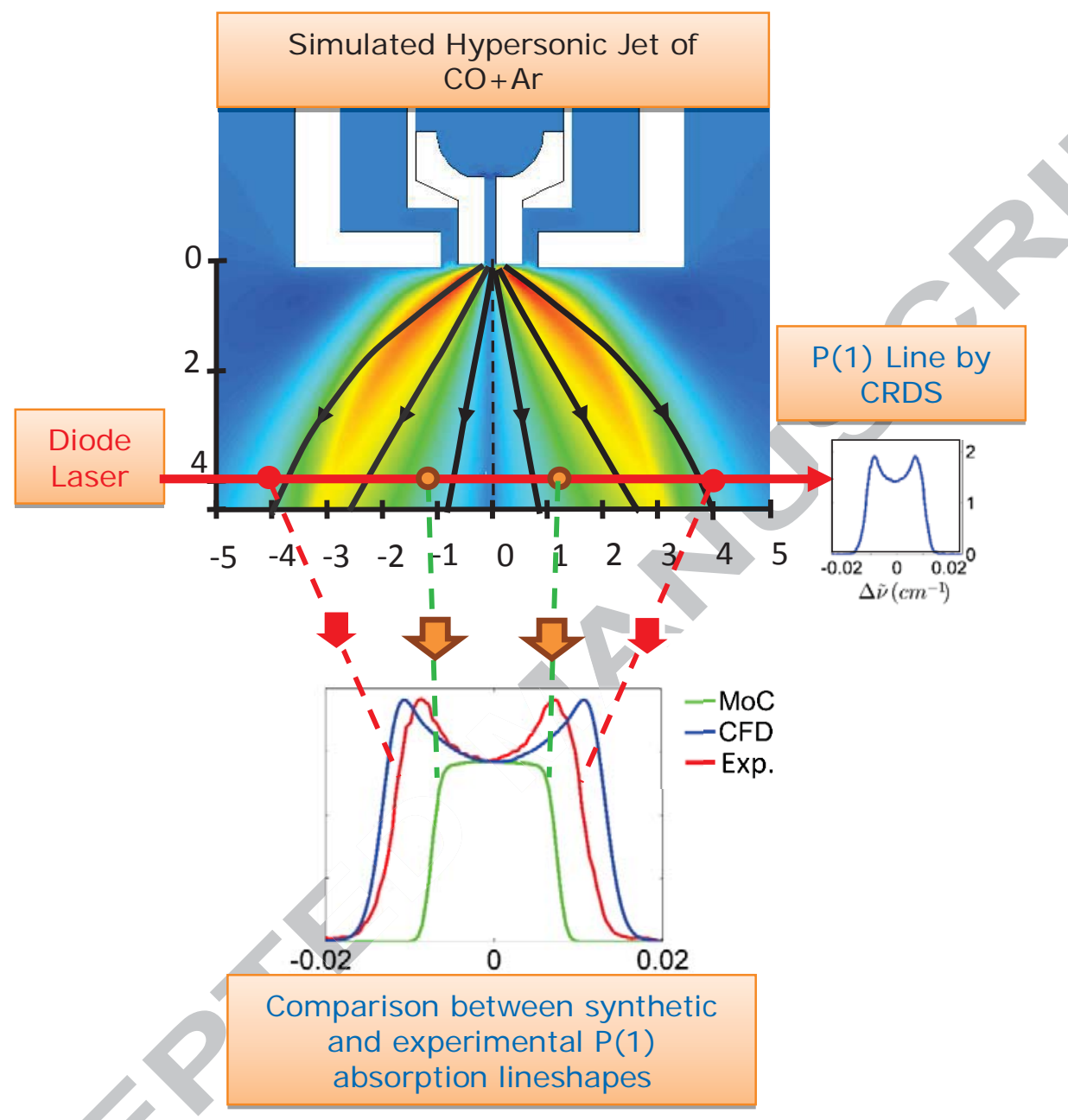


Highlights:

1. CRDS based line of sight measurements are carried out in hypersonic free-jet of carbon monoxide and argon.

2. A novel numerical technique is employed to obtain the synthetic line through interaction of laser with hypersonic flow.

3. During comparison, experimental and synthetic lines showed double peaked structure.

4. Contribution of each region of the jet is evaluated in building the integrated absorption line.

5. Process of integration revealed that retarded warmer non-isentropic region of the jet is responsible for double peaked line shapes. 